\title{
Efecto de la adición de materiales compuestos de estireno-butadieno/plumas de pollo en la modificación de asfaltos
}

\section{Efecto de la mezcla de materiales compuestos de estireno-butadieno / plumas de pollo en la modificación de asfaltos}

RAMOS-GALVÁN, Claudia Esmeralda†*, RIVERA-ARMENTA, José Luis, SALAZAR-CRUZ, Beatriz Adriana y ESCOBAR-MEDINA, Francisco Javier

Instituto Tecnológico de Ciudad Madero /Tecnológico Nacional de México

ID 1 ${ }^{\mathrm{er}}$ Autor: Claudia Esmeralda, Ramos-Galván / ORC ID: 0000-0002-6727-006X, CVU CONACYT ID: 25098

ID $1^{\text {er }}$ Coautor: José Luis, Rivera-Armenta / ORC ID: 0000-0002-9076-2353, Researcher ID Thomson: C-4080-2017, CVU CONACYT ID: 121513

ID $2^{\text {do }}$ Coautor: Beatriz Adriana, Salazar Cruz / ORC ID: 0000-0002-9466-2165, CVU CONACYT ID: 209015

ID $3^{\text {er }}$ Coautor: Francisco Javier, Escobar Medina

DOI: $10.35429 / J C E .2019 .9 .3 .1 .6$

Recibido 03 de Junio, 2019; Aceptado 20 Septiembre, 2019

\begin{abstract}
Resumen
El asfalto es un material importante para la construcción de carreteras y otras aplicaciones. Es por ello que se realiza la búsqueda de materiales que en combinación con el asfalto generen un producto de mejor calidad, menores impactos ambientales y bajos costos, permitiendo así verse reflejado mayor resistencia del asfalto a las condiciones climáticas extremas y al tránsito. Para cubrir estas diferentes necesidades, debe contarse con asfaltos con un mejor desempeño, teniendo que modificar sus propiedades químicas, físicas y reológicas, con el fin de mejorar su comportamiento al esfuerzo cortante, deformación, fatiga, envejecimiento, elasticidad, etc. En este trabajo se estudió el efecto de añadir materiales compuestos con polímero del tipo SBS lineal (estireno-butadieno-estireno) y la pluma de pollo, 1,3 y 5 PHR (partes por cien de hule) en proporciones de 2, 4 y $6 \%$ en relación al peso del asfalto. $\mathrm{Se}$ aplicaron técnicas para caracterizar tales como viscosidad dinámica, punto de reblandecimiento, caracterización por separación de fases, índice de penetración y microscopía fluorescente. Como resultado el utilizar un polímero del tipo SBS lineal incorporándole queratina de la pluma de pollo como refuerzo para el asfalto no se presenta resultados que pueden ser interesantes en áreas donde se requiera mayor fluidez.
\end{abstract}

Asfalto, Pluma de pollo, Polímero SBS lineal

\begin{abstract}
Asphalt is an important material for road construction and other applications. That is why it is done the search for materials that in combination with the asphalt generate a product of better quality, lower environmental impacts and low costs, thus allowing to be reflected greater resistance of the asphalt to the extreme climatic conditions and traffic. To cover these different needs, asphalts with a better performance must be counted, having to modify their chemical, physical and rheological properties, in order to improve their behavior to the shearing, deformation, fatigue, ageing, elasticity, etc. In this work, studied the effect of adding composite materials with polymer of the type SBS lineal (Styrene-butadienestyrene) and the feather of chicken, taking concentrations of 1, 3 and 5 PHR (parts per hundred of rubber) in ratios of 2, 4 and $6 \%$ in relation to the weight of asphalt. Techniques were applied to characterize such as dynamic viscosity, softening point, phase separation characterization, penetration index and fluorescent microscopy. As a result, using a polymer of the linear SBS type incorporating keratin from the chicken plume as reinforcement for the asphalt does not present interesting results for application areas such as where high fluidity is required.
\end{abstract}

Asphalt, Feather of chicken, Polymer SBS lineal

Citación: RAMOS-GALVÁN, Claudia Esmeralda, RIVERA-ARMENTA, José Luis, SALAZAR-CRUZ, Beatriz Adriana y ESCOBAR-MEDINA, Francisco Javier. Efecto de la adición de materiales compuestos de estireno-butadieno/plumas de pollo en la modificación de asfaltos. Revista de Ingeniería Civil. 2019. 3-9: 1-6

\footnotetext{
* Correspondencia del Autor (correo electrónico: cesmeralda@ hotmail.com)

$\dagger$ Investigador contribuyendo como primer autor.
} 


\section{Introducción}

El uso de asfaltos modificados en la construcción de pavimentos flexibles se ha extendido a nivel mundial como respuesta a condiciones más severas durante su vida útil y buscando un mejor desempeño que permita prolongar su duración. La modificación de asfaltos con polímeros busca mejorar sus propiedades viscoelásticas para cumplir con los requerimientos específicos del material; de manera muy concisa se puede decir que mediante la modificación se desea mejorar la elasticidad y la flexibilidad, la consistencia y la durabilidad con el fin de evitar que una mezcla asfáltica ante los cambios climatológicos y cargas a que están expuestos los pavimentos se fisure, se deforme o se desprenda.

En estudios recientes los polímeros p. ej. hule sintético estireno-butadieno (SBR), copolimero estireno-butadieno-estireno (SBS), etilen vinil acetato (EVA), polipropileno atactico (APP), etilen-glicilacrilato (EGA) han sido usados en la modificación de asfaltos para pavimentos, estudiándose las propiedades reológicas de los mismos y relacionándolos con la morfología.

Se sabe a partir de muchos estudios que los copolímeros de estireno-butadieno-estireno, son los materiales que mejor actúan como agentes modificantes del asfalto. Poseen los requerimientos estructurales óptimos para modificar efectivamente al asfalto; la característica primordial que deben poseer es que faciliten la formación de estructuras reticuladas o redes que atrapen fácilmente las moléculas de asfalto para proporcionarle mayor rigidez y con ello se eviten problemas como deformaciones y agrietamientos.

La mala disposición de los desechos sólidos de los recursos naturales que afectan adversamente el medio ambiente ha desarrollado un interés relacionado con el desarrollo de polímeros biodegradables para obtener compuestos mejorados, no solo por razones ambientales sino también por sus propiedades y sostenibilidad. La industria avícola genera más de cinco millones de toneladas de plumas de pollo cada año en todo el mundo. La queratina es una proteína presente en la pluma de pollo es el componente estructural, tiene varias funciones, incluyendo el control de temperatura y protección física o química, y también proporciona resistencia mecánica y elasticidad.
Se considera material de desecho y puede ser aprovechada en conjunto con un polímero para mejorar notablemente propiedades de otros materiales. Los materiales compuestos han tomado importancia dentro de la modificación de asfaltos. Estos se realizan por la combinación de dos o más materiales diferentes. Puede presentar propiedades mecánicas y físicas con mejores propiedades ya que las combina de sus componentes originales. Suelen elaborarse con una matriz polimérica la mayor parte se utilizan elastómeros y plastómeros con un agente modificador extra como fibras naturales.

Una opción viable es elaborar materiales compuestos utilizando plumas de pollo. La queratina es el componente principal de la pluma el cual tiene propiedades extraordinarias, es un material liviano con alta resistencia mecánica y térmica.

Ante las situaciones mencionadas, en este trabajo de investigación se estudia el efecto de añadir al asfalto un polímero del tipo lineal SBS (estireno-butadieno-estireno) con la pluma de pollo, llevándose a distintas concentraciones y se utilizan técnicas para caracterizar tales como viscosidad dinámica, punto de reblandecimiento, separación de fases e índice de penetración. Esto con el fin de obtener una mejor mezcla asfáltica que presente propiedades adecuadas.

\section{Metodología Experimental}

\section{Obtención de queratina de origen natural}

Las plumas de pollo se obtuvieron de un rastro local en Altamira, Tamaulipas. Se sometieron a lavado con agua y secarón al sol dos días. El raquis se separó de las barbas de la pluma. Se cortó el raquis en pequeños trozos que posteriormente fueron molidos en una pica lica T-fal en tiempos de $45 \mathrm{~s}$. Finalmente el polvo se tamizó en una malla tamaño 25 y con un tamaño de partícula de 707 micrones

\section{Preparación de compositos}

Se prepararon materiales compuestos con polímero SBS lineal y queratina a partir de la pluma de pollo en concentraciones de $1 \mathrm{phr}, 3 \mathrm{phr}$ y 5phr, (SBS/QX-phr) La obtención de los materiales compuestos se realizó en un equipo con cámara de mezclado intelli-Torque PlastiCorder/reómetro marca C.W.

RAMOS-GALVÁN, Claudia Esmeralda, RIVERA-ARMENTA, José Luis, SALAZAR-CRUZ, Beatriz Adriana y ESCOBAR-MEDINA, Francisco Javier. Efecto de la adición de materiales compuestos de estireno-butadieno/plumas de pollo en la modificación de asfaltos Revista de Ingeniería Civil. 2019 
Brabender Instruments PL2000, se llevó a cabo el mezclado a temperatura de $130^{\circ} \mathrm{C}$ con velocidad de $70 \mathrm{rpm}$. Los productos generados en la cámara de mezclado se llevaron a una prensa hidráulica marca Dake para la elaboración de las placas. Este equipo se operó a $350^{\circ} \mathrm{F}$, con tres diferentes presiones y cada presión con su respectivo tiempo, se empezó con una presión de 0 toneladas en un tiempo de 20 minutos, posteriormente una presión de 5 toneladas con un tiempo de 10 minutos y finalmente una presión de 8 toneladas con un tiempo de 8 minutos. El espesor de las placas fue de $1 / 8$ ".

\section{Preparación de mezclas asfálticas}

Para preparar las mezclas modificadas primero se precalentó el bloque metálico durante 30 minutos, las mezclas se realizaron en un periodo de 120 min, este tiempo se estableció por pruebas previas hasta observar visualmente que no existiera material polimérico en la mezcla, el compuesto fue respectivamente añadido a una relación del $2 \%, 4 \%$ y $6 \%$ en peso del asfalto. En este proceso se mantuvo un control de temperatura a $190^{\circ} \mathrm{C}$ con un desfase $\pm 2{ }^{\circ} \mathrm{C} \mathrm{y}$ velocidad de $1200 \mathrm{rpm}$ para logar la mejor dispersión posible, en la tabla 1 se observa la matriz experimental..

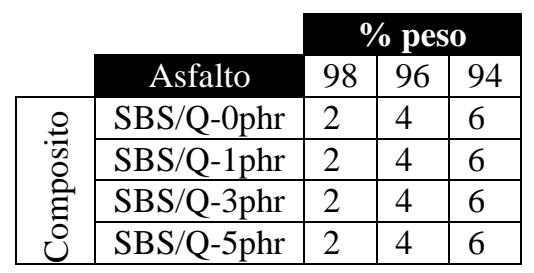

Tabla 1 Matriz experimental de muestras SBS lineal/pluma de pollo

\section{Caracterización}

Se determinó la viscosidad dinámica mediante un viscosímetro brookfield modelo DV-II+ Pro conectado a una cámara termostatizada, de acuerdo a la norma se utilizarón $10.5 \mathrm{~g}$ de mezcla asfáltica. Se usó la prueba ASTM D36 de anillo y bola $(\mathrm{R} \& \mathrm{~B})$ para determinar el punto de ablandamiento del asfalto. Para la separación de fases se utilizó un método que consiste en observar la estabilidad de compatibilidad de un polímero en el asfalto mediante una muestra que se sujeta a altas temperaturas durante un tiempo y después se coloca en un congelador, se toma como referencia la norma ASTM D5892 en donde se vertieron $50 \mathrm{~g}$ de muestra asfáltica.
La prueba de penetración ASTM D5-86 se llevó a cabo utilizando un penetrómetro Koeler K95500. Para determinar el nivel de dispersión y compatibilidad entre los componentes de las mezclas asfálticas se realizó mediante la técnica de microscopía de fluorescencia, en un Carl Zeiss Modelo Axio Tech n/s 180935 óptica: 20x.

\section{Resultados}

\section{Viscosidad dinámica}

Para la proporción al 2\% (Figura 1) se observa que la mezcla SBS/Q0-phr-2\% presenta valores altos de viscosidad, debido a que por ser puro polímero la interacción entre moléculas que contienen los dobles enlaces que presenta el butadieno con misma fuerza molecular hace que se atraigan y tengan una mayor cohesión molecular generando así una mezcla con menor movimiento entre moléculas. Cuando se añade queratina en las mezclas SBS/Q1-phr-2\%, SBS/Q3-phr-2\% y SBS/Q5-phr-2\% se provoca un efecto que suaviza la mezcla asfáltica generando una disminución en la viscosidad.

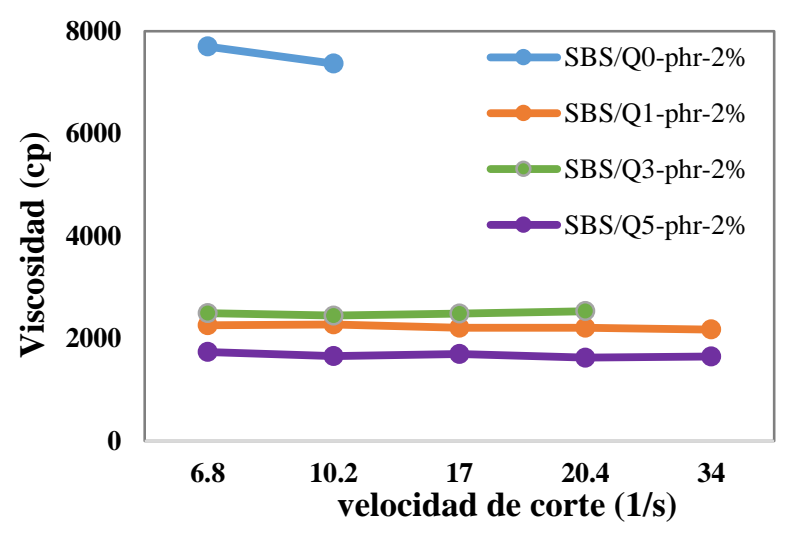

Figura 1 Viscosidades de mezclas asfálticas al $2 \%$ a $135^{\circ} \mathrm{C}$

En la figura 2 se observa que aumenta la viscosidad para la mezcla SBS/Q5-phr-4\% debido a que por haber mayor cantidad de composito aumenta la viscosidad pero conforme aumenta la temperatura no hay un cambio significativo. Para las mezclas preparadas al 6\% se nota un efecto de saturación porque vuelven a presentar valores de viscosidad similares a las del $2 \%$. 


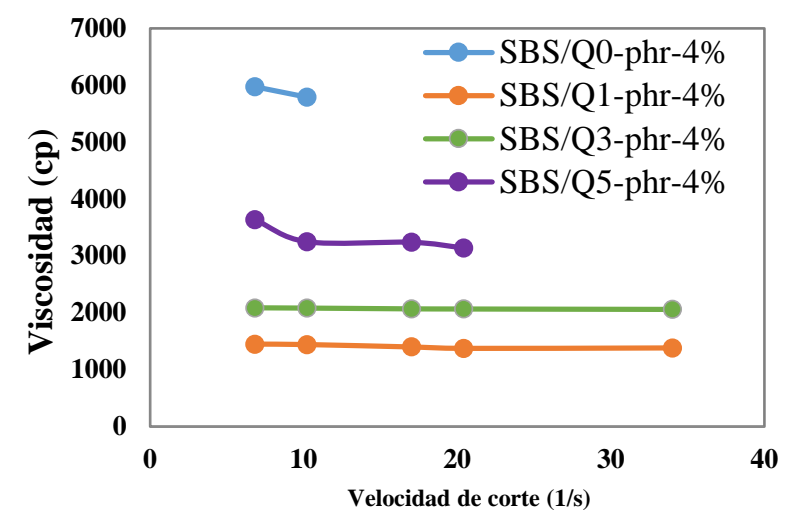

Figura 2 Viscosidades de mezclas asfálticas al $4 \%$ a $135^{\circ} \mathrm{C}$

\section{Punto de reblandecimiento}

En la figura 3 se observa que las mezclas que presentan el valor más alto son los compuestos con puro polímero. Se puede observar que la relación de adicionar el polímero al 2, 4 y $6 \%$ el parámetro de R\&B es muy similar y no afecta significativamente agregar mayor cantidad de polímero para mejorar la propiedad. Por otro lado la adición de queratina causa un efecto de disminución a la propiedad al momento de añadir $1 \mathrm{phr}$ y se ve un comportamiento de aumento hasta alcanzar $3 \mathrm{phr}$.

Las mezclas preparadas a $3 \mathrm{phr}$ se pueden considerar que sobresalen en comparación con los demás compuestos con queratina con una ligera mejora en la propiedad, esto se puede atribuir a que la queratina al estar en esa cantidad en equilibrio con el asfalto le proporciona un mayor tiempo de duración de carga ya que es un material que proporciona buena resistencia mecánica pero posterior a ello hay una saturación. Con lo anteriormente mencionado añadir refuerzo de queratina obtenida de la pluma de pollo junto con polímero SBS del tipo lineal no se nota un efecto mayor en contraste con los blancos de referencia.

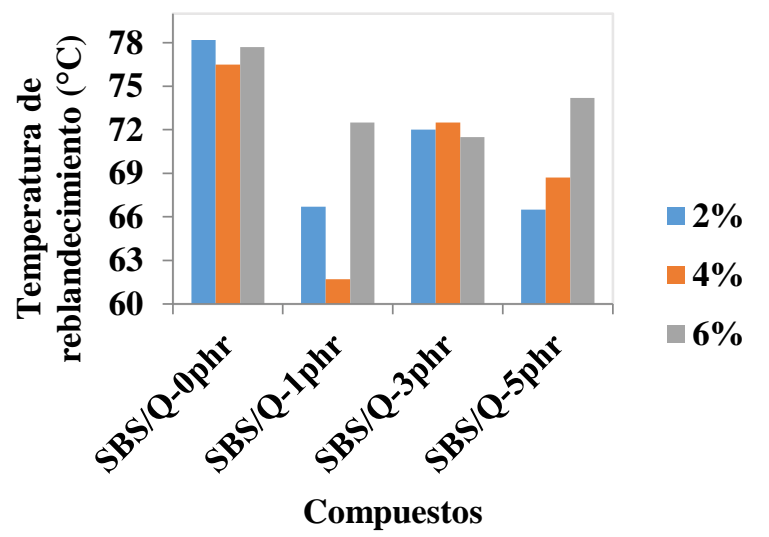

Figura 2 Análisis de datos prueba punto de reblandecimiento

\section{Separación de fases}

En la figura 4 se observa que los compuestos con queratina presentaron separación de fases al obtener valores altos de temperatura tomando en cuenta que si logra pasar el TRB $\geq 3^{\circ} \mathrm{C}$ para esta prueba se considera que la mezcla asfáltica es inestable. Los compuestos con puro polímero presentan un mejor valor que los compuestos con queratina y el efecto de agregar más cantidad de polímero no afecta significativamente al asfalto. Para los compuestos en proporción al $2 \%$ de compuesto con queratina presentan una ligera mejora en la propiedad del asfalto más no favorable como se desea en menor valor. La presencia de la queratina en el hule aparentemente no favorece a la homogenización del asfalto, presentando mejor respuesta a medida que la cantida de queratina es más baja.

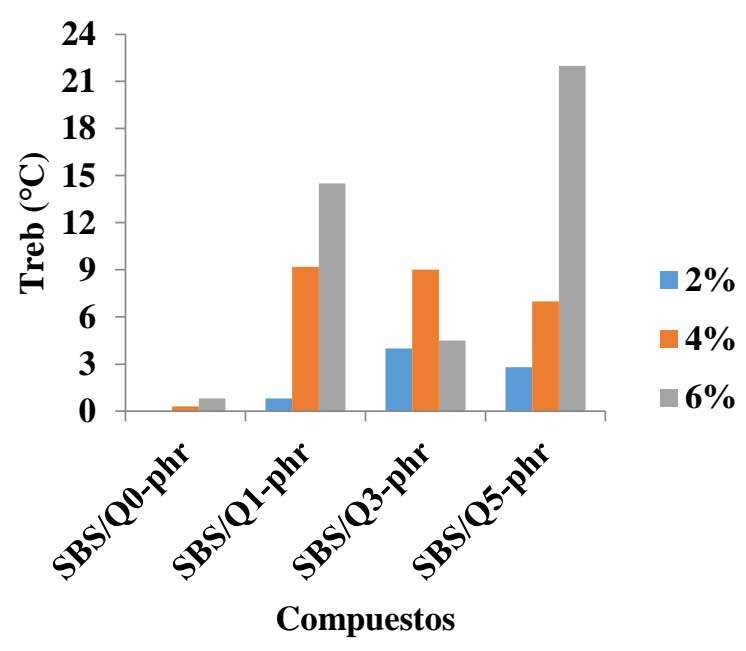

Figura 3 Análisis de datos prueba separación de fases

\section{Penetración}

En la figura 5 los blancos de referencia vuelven a presentar mayor contraste que los compuestos de queratina al tener menores valores de penetración. Cuando se adiciona compuesto al $2 \%$ de puro polímero el valor de penetración disminuye un 53\% en comparación con el asfalto virgen. Sin embargo agregar más polímero no mejora esta propiedad y al contrario se incrementa su valor de penetración. Se observa que a medida que se aumenta la concentración de compuesto con queratina se tiene mayores valores de índice de penetración. 
Este hecho se atribuye al generarse cadenas grandes por acción de los enlaces de azufre con el polímero se rompan y la cohesión molecular ya no es tan fuerte generando en las mezclas una menor rigidez en el asfalto y también por el tipo que se utiliza al presentar cadenas lineales favorece aún más esto porque presentan mayor movilidad. Este comportamiento no es adeucado si se considera la aplicación de los asfalto modificados en pavimentos, sin embargo existen otro tipo de aplicaciones en las cuales el adelgazamiento o que el material sea más suave pueden ser interesantes.

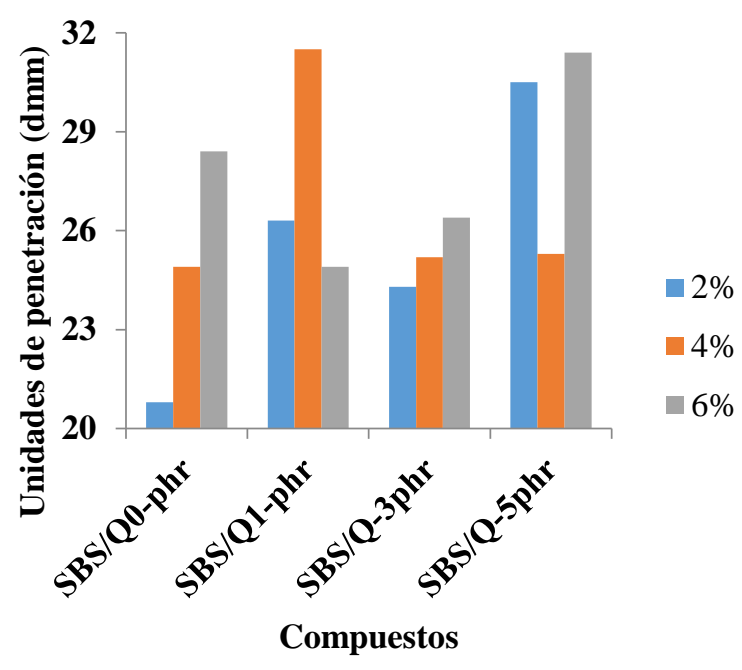

Figura 4 Consistencia de los asfaltos (Penetración)

\section{Microscopía fluorescente}

En las micrografías (Tabla 2), el fondo oscuro representa la fase asfáltica y los destellos de color están asociados al modificador, se debe tener en cuenta que las muestras que no presentan partículas fluorescentes indican una mejor dispersión del aditivo en el asfalto. Para las microscopias de SBS-Q0-phr al 2 y $4 \%$ no se identifica dispersión de material y por eso no incluyen y para la de $6 \%$ se ven pequeñas partes de polímero hinchado, esto se puede atribuir al hecho de que el tipo de polímero que se utiliza no logra dispersarse bien en el asfalto y al tener en su estructura cadenas lineales estas se mueven fácilmente donde no permitan formar una red estable dando así una mezcla heterogénea. Cuando se adiciona queratina a $1 \mathrm{phr}$ y $3 \mathrm{phr}$ se empieza a ver una mejor dispersión del polímero en el asfalto esto se debe que al haber queratina en el asfalto, los enlaces con azufre empiezan a unirse con los dobles enlaces del butadieno y le confieren un mejor ordenamiento en su estructura formando así una mezcla cada vez homogénea.
Con 5 phr y al agregar mayor cantidad de compuesto en proporción del asfalto se observa una saturación de polímero debido a que tanto el polímero como la fase malténica al no haber asfáltenos se reflejan zonas donde se produce una inversión de fases acumulándose polímero hinchado y huecos donde se ve parcialmente homogénea la mezcla asfáltica

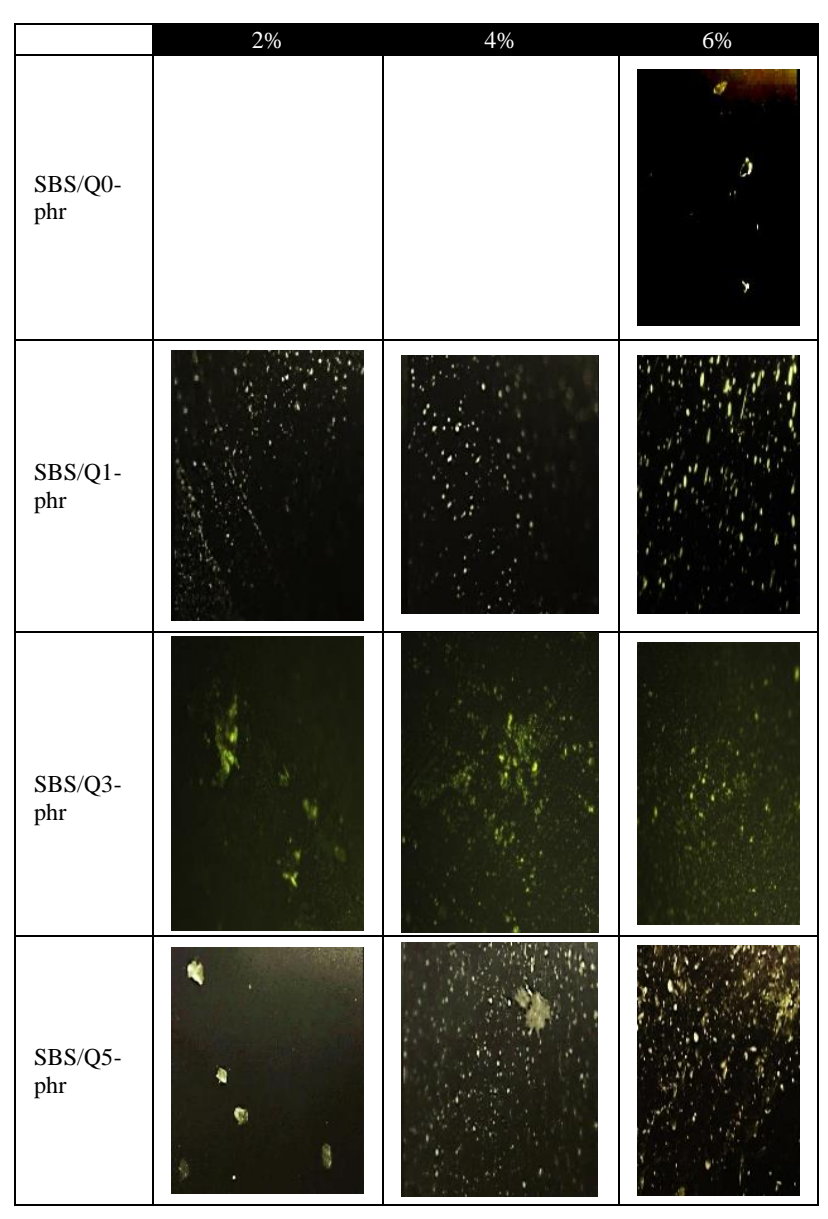

Tabla 1 Micrografías de fluorescencia de mezclas asfálticas

\section{Conclusiones}

De acuerdo con los resultados obtenidos utilizar un polímero del tipo SBS lineal incorporándole queratina como refuerzo para el asfalto no presenta un mayor desempeño, esto debido al tipo de polímero que se utiliza en este caso lineal al no tener una estructura tan reticulada como la de un tipo radial. La queratina al presentar en sus enlaces azufre y estar expuestos a un ambiente de temperatura alta le confiera al polímero lineal en su estructura un mayor grado de despliegue y esto haga un rompimiento entre las dobles ligaduras del hule polibutadieno con los policiclos del asfalto. 


\section{Agradecimiento}

Al Tecnológico Nacional de México (TeCNM) por el financiamiento con el proyecto "Preparación de asfaltos modificados utilizando pluma de pollo como agente modificante", con clave 6687.18-P

\section{Referencias}

Caro S., Vega N., Husserl J. \& Alvarez A.. (2016). Studying the impact of biomodifiers produced from agroindustrial wastes on asphalt binders. Construction and Building Materials. Vol. 126, 369-380.

Chen C., Podolsky J., Williams C. \& Cochran E. (2018). Laboratory investigation of using acrylated epoxidized soybean oil (AESO) for asphalt modification. Construction and building materials. Vol. 187, 267-279.

Quintero-Curvelo, G.A., Huertas-Díaz E., Ortega-David, E. (2017). Procesamiento de plumas de pollo para la obtención de queratina. UGCiencia, 23, 81-87

Ran Zhang, Hainian Wang, Junfeng Gao, Zhanping You \& Xu Yang. (2017). High temperature performance of SBS modified bioasphalt. Construction and Building Materials. 100-105.

Sánchez Miranda, N. (2016). Pavimentos asfalticos de alto modulo (Caso de estudio; libramiento Dr. González, N.L.). México, D.F.: ESIA.

Zapién-Castillo, S., Rivera-Armenta, J. L., Chávez- Cinco, M. Y., Salazar-Cruz, B.A. \& Mendoza-Martínez, A. M. (2016). Physical and rheological properties of asphalt modified with SEBS/montmorillonite nanocomposite. Construction and Building Materials. Vol. 106, 349-356. 